\title{
Éditorial. Les médias des minorités ethniques. Représenter l'identité collective sur la scène publique
}

Ethnic minority media. Representing collective identity on the public sphere

Les medios de comunicación de minorías étnicas. Representar la identidad en la escena pública

Isabelle Rigoni

\section{(2) OpenEdition}

Journals

Édition électronique

URL : https://journals.openedition.org/remi/5027

DOI : $10.4000 /$ remi.5027

ISSN : $1777-5418$

Éditeur

Université de Poitiers

Édition imprimée

Date de publication : 1 février 2010

Pagination : 7-16

ISBN : 978-2-911627-54-5

ISSN : 0765-0752

Référence électronique

Isabelle Rigoni, «Éditorial. Les médias des minorités ethniques. Représenter l'identité collective sur la scène publique », Revue européenne des migrations internationales [En ligne], vol. 26 - n 1 | 2010, mis en ligne le 01 février 2013, consulté le 14 avril 2022. URL : http://journals.openedition.org/remi/5027 ; DOI : https://doi.org/10.4000/remi.5027 


\section{Éditorial}

\section{Les médias des minorités ethniques. Représenter l'identité collective sur la scène publique.}

\section{Isabelle RIGONI*}

Nous proposons dans ce dossier d'explorer les initiatives médiatiques de populations en situation de minorisation ethnique (Simon, 2006). Dans la lignée des recherches sur les « ethnic media » (Titley, 2008 ; Georgiou, 2006 ; Husband, 1994 ; Cottle, 2001), et notamment de celles menées par les chercheurs de l'équipe européenne MinORITYMEDIA ${ }^{1}$, les articles ici réunis sont consacrés aux médias créés par et pour les populations migrantes et leurs enfants, mais aussi par des populations qui partagent une mémoire de la mobilité, à l'instar des Roms. Produits de la migration mondialisée (Simon, 2008), de l'affirmation des cultures minoritaires et des rapports post-coloniaux, les médias des minorités ethniques sont à la fois des supports et des producteurs d'identités. Bien que contemporains des grands courants migratoires depuis la seconde moitié du XIX ${ }^{\mathrm{e}}$ siècle, ils ont vu leur rôle réévalué avec la révolution technologique en matière d'information et de communication. En ce sens, leur apport est devenu fondamental dans la compréhension des logiques de mise en représentation du soi collectif et de mobilisations identitaires. Il s'agit dans ce dossier de comprendre le rôle de la communication dans les représentations des identités collectives - et tout particulièrement de souligner celui des médias des minorités ethniques en termes de production et de représentation identitaires.

\section{UN PHÉNOMÈNE ANCIEN, CONCOMITANT DES PREMIÈRES VAGUES MIGRATOIRES}

Forts de plusieurs milliers de titres dans les démocraties occidentales ${ }^{2}$, les médias des minorités ethniques représentent un phénomène important et histori-

* Chercheure, responsable de l'équipe européenne MinORITYMEDIA, MSHS-MIGRINTER, 99, avenue du Recteur Pineau, 86000 Poitiers, isabelle.rigoni@univ-poitiers.fr

1 MinORITYMEDia est une équipe européenne d'excellence financée dans le cadre du $6^{\mathrm{e}}$ PCRDT de la Commission européenne, accueillie à l'Université de Poitiers et hébergée à MIGRINTER. La recherche quadriennale (2006-2010), coordonnée par Isabelle Rigoni, porte sur les médias des minorités ethniques dans 8 pays d'Europe (Allemagne, Espagne, France, Hongrie, Italie, Pays-Bas, Royaume-Uni, Turquie) : www.minoritymedia.eu

2 L'équipe MinORITYMedia a recensé en 2007 plus de 5000 médias dans les 8 pays européens de son étude de terrain. Aux États-Unis, the American Newspaper Publishers Association 
quement ancré. Les premiers titres apparaissent dès le milieu du XIX $\mathrm{X}^{\mathrm{e}}$ siècle, avec l'arrivée des Arméniens en France, suivis d'une presse belge, italienne et polonaise. Robert Ezra Park, dès 1922, observe des initiatives semblables de la part d'immigrants à Chicago (Park, 1970, 2008). Pourtant et malgré leur ancienneté, les médias des minorités ethniques demeurent encore mal connus. En 1994, Charles Husband lançait un appel pressant pour que « les énergies de la recherche soient dirigées vers l'examen de la situation des minorités ethniques comme agents actifs de la production médiatique ». Quelques années plus tard, Simon Cottle déplorait lui aussi ce manque d'intérêt : "Studies of ethnic minority audiences, remarkably, remain a rarity. Given the recent enthusiasm for ideas of 'active' audiences in recent media approaches, this silence, with a few exceptions only, is perhaps all the more surprising. In another sense, however, it simply continues the institutional logic and academic inertia which, until recently, has conspired to ignore what ethnic minorities themselves may think, want, or say about media representations, the media's involvement within their everyday lives, or their media hopes for the future » (Cottle, 2000 : 23-24). Dans les années 1990, ce sont des chercheurs anglo-saxons et scandinaves qui les premiers vont utiliser les termes d' " ethnic media » ou d' « ethnic minority media » pour qualifier ces phénomènes. Le plus souvent analysés dans le cadre des théories habermassiennes de la sphère publique (Husband, 1994, 2000), les travaux des chercheurs mobilisent aussi les notions d'inclusion et d'exclusion (Georgiou et alii, 2007) et de citoyenneté.

Plus récemment sociologues et historiens montrent que c'est dans un contexte de mal-représentation et de sur-stigmatisation (Rigoni 2008, 2007a, 2007b) que nombre de personnes en situation de minorisation se tournent vers des médias « alternatifs » qui leur semblent mieux répondre à leurs besoins d'information de façon générale. Depuis le milieu des années 1990, les recherches se sont essentiellement focalisé sur les télévisions satellitaires et sur la multiplication concomittante des paraboles (Mattelart, 2007, 2002 ; Guaaybess, 2005 ; Lamloum, 2004). Et hormis dans l'aire anglo-saxonne, peu d'entre elles se sont intéressées à la production et à la réception des médias des minorités ethniques.

Pourtant, l'appréhension des médias produits par et pour les minorités ethniques donne à voir un paysage très diversifié. Jean-Paul Marthoz (2001) souligne combien « la domination que semblent exercer quelques grands médias » fait écran à « l'extraordinaire complexité et diversité de l'univers médiatique » que dessinent ces médias désignés tour à tour comme « minoritaires », " issus de l'immigration », « ethniques », « communautaires », « de la diversité » (Rigoni, 2010)... Variété d'appellations qui renvoie à l'extrême hétérogénéité des situations qu'ils recouvrent, de la télévision commerciale nationale (Naficy, 1993 ; Ben Amor-Mathieu, 2000) aux journaux ou émissions associatifs locaux (Downing \& Husband, 2005). Très récemment, avec la multiplication des médias électroniques, de plus en plus de recherches, notamment au sud et à l'est de l'Europe, se sont consacrées aux productions médiatiques ethniques ${ }^{3}$.

estimait en 1990 que les minorités ethniques composaient $18 \%$ de l'industrie de presse et $16 \%$ de la force de travail.

3 Le colloque "Se représenter autrement? Les médias des minorités ethniques, entre hégémonie et résistances » organisé en mars 2010 par MIGRINTER à l'occasion de la clôture 


\section{RENOUVELLEMENT DES OUTILS DE COMMUNICATION ET LOGIQUES IDENTITAIRES}

La pérennisation des médias des minorités ethniques est contemporaine de modes renouvelés d'affirmations identitaires, de mobilisations transnationales et de visibilité des minorités culturelles et religieuses. Les mobilisations identitaires ne constituent pas une tendance nouvelle, mais s'inscrivent plus largement, pour la période contemporaine, dans le prolongement des mouvements culturels - femmes, homosexuels, régionalistes - qui ont marqué les années 1970 du sceau d'une affirmation identitaire qui a pu être analysée, déjà, comme participant à la fragmentation des identités nationales (Wieviorka, 1996). En revanche, leurs modalités pratiques et, surtout, leurs outils sont inédits : la popularisation des technologies de l'information et de la communication (TICs) a permis la diffusion massive d'une multitude de représentations identitaires individuelles et collectives, sur les modes culturels et religieux. Les migrants et les minorités culturelles et religieuses ont, parmi les premiers, utilisé les outils communicationnels numériques (Blondeau, 2007). La percée des TICs dans les années 1980, puis la croissance rapide de l' «Internet social » (blogs, wikis, sites de réseaux sociaux) (Allard, 2005) dans les années 2000 ont bouleversé les rapports à l'espace-temps en même temps qu'elles ont favorisé l'émergence de nouvelles pratiques sociales et communautaires. L'éclatement de la bulle technologique, qui affecte les secteurs liés à l'informatique et aux télécommunications et dont l'apogée a lieu en mars 2000, n'affecte pas la consommation médiatique grand public qui, bien au contraire, prend au même moment son véritable essor (Charon, 2003). Tout au long des années 2000, le taux d'équipement et le taux d'utilisation d'Internet ne cesse de croître dans les foyers européens. Les internautes à domicile sont de mieux en mieux équipés, la connexion en haut débit devenant la norme dans l'immense majorité des foyers occidentaux. Immigrés, minorités ethniques et diasporas sont parmi les premiers bénéficiaires des progrès technologiques en matière d'information et de communication qui facilitent les échanges matériels et immatériels avec le pays d'origine ou entre plusieurs espaces migratoires. Le recensement des médias des minorités ethniques effectué par l'équipe européenne MiNORITYMEDiA indique que les premiers médias électroniques ethniques apparaissent dès le milieu des années 1980 en Grande-Bretagne, en Allemagne et en France, à un moment où l'utilisation d'Internet demeure encore confidentielle et où très peu de foyers sont équipés d'un ordinateur et abonnés à un fournisseur d'accès. C'est toutefois véritablement à partir de l'année 2000 que les minorités ethniques profitent de l'élan permis par l'arrivée massive des technologies Internet pour investir le web. Dès lors, webzines, web-radios et web-TVs à caractère ethnique se multiplient. La popularisation d'Internet gagne désormais toutes les franges de la société et nombre de minorités ethniques s'approprient les multiples avantages de ce média dématérialisé et à vocation transnationale.

du programme de recherche MiNORITYMEDIA, démontre l'intérêt grandissant au niveau international pour l'étude des médias des minorités ethniques. L'appel à communications a donné lieu à 68 propositions issues de 22 pays, tandis que le site web www.minoritymedia.eu a été consulté durant les 6 semaines de l'ouverture de l'appel dans 50 pays sur tous les continents, selon les statistiques de Google Analytics. 
La diversification des moyens de communication affecte au premier plan les rapports migratoires et les relations diasporiques. Ces nouveaux modes de communication ont été interprétés comme le déclencheur de la mise en place d'une "société en réseau » qui laisserait place à une «modernité liquide » (liquid modernity) (Bauman, 2000) ou encore à une "seconde modernité » (second modernity) (Beck, 1992). L'intensification de l'utilisation des TICs et l'avènement du Web 2.0 (participatif, interactif) ont permis une plus grande expressivité des acteurs culturels et religieux, en introduisant de nouvelles facilités d'accès et de nouveaux moyens de communication. L'année 2005 reflète cette évolution, avec la naissance de milliers de blogs et de forums dédiés aux questions culturelles et religieuses. La mutation de l'Internet, devenu une plateforme communicationnelle et non plus spécifiquement informationnelle comme à ses débuts, facilite la création, la mise en lien et la redistribution des ressources, des productions et des opinions des internautes. Les minorités ethniques sont particulièrement impliquées dans l'utilisation du Web 2.0 en raison des modalités simples, démocratiques et ouvertes de la circulation de l'information. La construction et l'alimentation de réseaux de contacts de diverses natures (familiaux, amicaux, intimes, inconnus...) se complexifient à mesure de l'utilisation des applications du Web 2.0. Les « cyberminorités », « cyberdiasporas » et « cybermigrants » expérimenteraient à travers lui une «extraversion culturelle » (Hassane, 2009). Christian Licoppe (2002) évoque la "présence connectée » entre les membres géographiquement dispersés d'une même famille, Dana Diminescu (2007) met en avant la figure du « migrant connecté », Mihaela Nedelcu (2009) celle du « migrant online » et Serge Proulx (2008) celle du « nomade connecté ». Des recherches sociologiques sur les médias électroniques des diasporas (Mattelart, 2009) ainsi que des minorités ethniques (Georgiou et alii, 2007 ; Georgiou 2006) et religieuses (Siapera, 2006a, 2006b, 2007) - notamment sur la participation de ces minorités à la blogosphère (Siapera 2009) - montrent que nous assistons à l'émergence de nouvelles formes du vivre ensemble en même temps qu'à de nouvelles formes de représentations identitaires. Les liens forts et les liens faibles, décrits par l'analyste des réseaux sociaux Mark Granovetter (1973), sont réorganisés dans une temporalité de l'immédiateté qui contribue à la redéfinition du fonctionnement des rapports sociaux. L'Internet est devenu, pour nombre de minorités ethniques, un moyen de représentation communautaire et un mode d'intervention dans le débat public.

Dans un tel contexte, comment appréhender le rôle joué par les outils et les espaces communicationnels dans les logiques identitaires des populations minorisées dans l'espace public ? L'enjeu de ce dossier revient notamment à comprendre les logiques de la médiation de l'appartenance identitaire ou, en d'autres termes, de ce que nous appelons les identités médiées - identités qui se (re)produisent et se donnent à voir au contact et au moyen des médias. Alors que l'on constate que la communication médiatique joue un rôle de plus en plus central dans le quotidien des minorités ethniques, l'objectif des recherches est de décrypter, au prisme de la production médiatique, la complexité des affiliations identitaires et les processus de (re)construction communautaire. Il s'agit de comprendre comment se manifestent les appartenances, la représentation d'un soi collectif, et enfin les expressions et pratiques culturelles que permettent les nouveaux médias (webzines d'information et/ou d'opinion, blogs, réseaux sociaux) et les médias traditionnels (presse écrite, radio, télévision) des minorités ethniques. 
Ce dossier présente une série de contributions rendant compte de recherches croisées sur les processus sociaux de mobilité (fait migratoire, fait diasporique, fait minoritaire) et sur le champ d'expérience des minorités ethniques dans le paysage médiatique qui leur est propre (échanges symboliques, mobilisations). Deux approches sont privilégiées : la mise en visibilité et en concurrence des offres identitaires d'une part, la complexité des relations multi-spatiales d'autre part.

\section{VISIBILITÉ ET CONCURRENCE DES OFFRES IDENTITAIRES...}

Les médias des minorités ethniques réalisent un double travail : favoriser l'intégration de leurs audiences à la société dominante, que cette intégration soit civique, politique ou économique ; et construire et homogénéiser la "communauté » dont ils dépendent. Ces médias participent d'un espace public où se manifeste et se reconstitue une offre d'identités collectives à même de susciter ou de renforcer l'adhésion à des «communautés imaginées ». Ces identités médiées sont des identités de flux, en (re)construction permanente, véhiculées et (re)produites par les médias qui les portent. Les médias des minorités ethniques servent notamment de caisse de résonance aux luttes d'auto-définition de certains groupes. Les représentants et porteparole de ces minorités sont mis en visibilité mais aussi en concurrence, et les médias tendent à favoriser l'apparition de nouveaux supra-interlocuteurs, qu'ils légitiment en leur donnant un espace d'expression.

Dédié aux enjeux de la « re-présentation », l'article de Claire Cossée montre bien comment les « artisans des médias tsiganes » en France et en Hongrie sont aussi des interlocuteurs des autorités politiques, contribuant notamment ainsi à « renverser le déni du droit à s'autodéfinir dont les Tsiganes font généralement l'objet ». L'auteure dégage trois courants médiatiques tsiganes en France, les médias religieux, ceux des professions ambulantes, et ceux issus du militantisme politique et en particulier syndicaliste. Ce paysage médiatique s'inscrit dans un schéma de concurrence pour la « re-présentation » du soi collectif et la prise de parole dans l'espace public. En Hongrie, Claire Cossée décrit la participation de plus en plus importante des intellectuelles roms dont l'investissement contribue à défier le mouvement tsigane à hégémonie masculine. L'auteure introduit ici une dimension centrale : la concurrence entre les acteurs de la « re-présentation » se donne également à voir dans les rapports de genre.

Dans sa typologie des sites Internet noirs en France, en Grande-Bretagne et aux États-Unis, Souley Hassane repère dix groupes qui se trouvent parfois en concurrence dans le travail de définition de l'identité. C'est le cas notamment de l'interprétation historique et de la construction de la mémoire collective dans son rapport à l'esclavage, à la domination économique et aux formes de discriminations ; c'est aussi le cas de l'inscription religieuse.

Retraçant la genèse de la presse nippo-brésilienne dès le début du $\mathrm{XX}^{\mathrm{e}}$ siècle, Helena Prado montre les tendances qui l'animent selon les différents courants migratoires du Japon vers le Brésil. La diffusion d'une identité nippo-brésilienne dans 
la presse nikkei n'est pas exempte de tensions, et provoque des questionnements parmi les générations successives d'immigrants quant à leur identification culturelle.

Martijn Oosterbaan, fin connaisseur des réseaux sociaux et des magazines diasporiques brésiliens, nous offre une analyse des courants et des enjeux identitaires qui les traversent. Fort de plusieurs millions de visiteurs brésiliens de par le monde, l'exemple du réseau social Orkut, qui compte des « communautés d'amis » fondées sur des sentiments de proximité (sociale, géographique, de classe, etc.), est particulièrement significatif de cette mise en concurrence des acteurs sociaux.

Kira Kosnick pour sa part fournit un panorama du paysage médiatique ethnique, particulièrement turcophone, en Allemagne. Celui-ci révèle les positions hégémoniques de certains acteurs médiatiques ainsi que le développement récent de stratégies commerciales (qui visent notamment à identifier des niches ethniques) au détriment des programmations multiculturelles des années 1990 qui défendaient une autre idée de la mixité sociale et culturelle.

\section{...ET COMPLEXITÉ DES RELATIONS MULTI-SPATIALES}

L'effort des médias des minorités ethniques à se faire reconnaître par les autorités locales, nationales voire supranationales montre que l'espace public est investi à différentes échelles spatiales et politiques. Les productions médiatiques sont à la fois dépendantes et créatrices de mobilisations translocales et transnationales. Dans cette logique, les différents articles discutent de la redéfinition de l'espace (concret autant que virtuel) et des frontières (matérielles et symboliques). Tandis que certains chercheurs s'interrogent sur la « fin des territoires » (Badie, 1995 ; Moreau Defarges, 1993), d'autres, repèrent de nouveaux « territoires circulatoires » (Tarrius, 1996) et de nouvelles logiques spatiales (Georgiou, 2006). Dès lors, se profile l'émergence d'un espace transnational inter-connecté, espace privilégié pour la production et la promotion de la «diversité culturelle ». Toutefois, ce dossier entend contribuer à démontrer que nous ne pouvons pas étudier les médias des minorités ethniques dans le contexte de la mondialisation et de la reconnaissance institutionnelle de la diversité sans interpréter la centralité des pratiques de communication (trans)nationales mais également (trans)locales et (trans)régionales. Les productions médiatiques expérimentent de nouvelles spatialités en interconnectant les dimensions locale et transnationale. Pour paraphraser Stuart Hall (1999), le local et le global sont désormais liés dans la mesure où chacun est la condition d'existence de l'autre. Une infrastructure médiatique mondialisée avec des réseaux de communication et des technologies diversifiés (Internet, satellite, numérique) alimente un nouvel espace public communicationnel qui façonne à son tour de nouvelles sphères publiques supra- et subnationales.

Myria Georgiou propose dans son article une réflexion sur les relations entre espace, identité et média. Elle se livre à une analyse multi-spatiale de l'appartenance diasporique et de sa traduction dans le champ médiatique. Dans la lignée des études sur le cosmopolitanisme, elle nous invite à (re)considérer les connexions dans l'espace 
comme des éléments clé de l'identité et du lien communautaire. En ce sens, le local, le national et le transnational forment une «matrice spatiale interconnectée » au sein de laquelle de nouvelles possibilités d'appartenance émergent. Myria Georgiou s'intéresse aux grands centres urbains mondialisés et, tout particulièrement, aux « ponts » qui les relient. Il s'agit de rendre intelligibles les productions culturelles médiées en tant que formes alternatives d'expression et d'auto-représentation.

C'est également cette urbanité mondialisée qui intéresse Kira Kosnick dans son article sur la réception des « médias minoritaires de masse » parmi les minorités ethniques et les « (post)migrants ». Elle constate que ce qui domine chez les auditeurs urbains est le sentiment de faire partie d'une « urbanité mondialisée » beaucoup plus que celui d'appartenir à une minorité ethnique. Dès lors, c'est la question de la relation entre les publics et l'«espace géographiquement localisable » qui est posée.

Martijn Oosterbaan centre aussi ses recherches sur les «villes globales » qui forment «les nœuds du réseau mondial de la finance et de l'information » et sur les utilisateurs des médias diasporiques brésiliens virtuels à Amsterdam et à Barcelone. Il tente de comprendre la façon dont des populations migrantes engagent et banalisent de nouveaux types de relations sociales (virtuelles) à travers l'espace. Pour cet auteur, le lieu géographique, physique, demeure un facteur essentiel dans le processus de construction et de partage de l'identité sociale de ces communautés migrantes virtuelles.

Claire Cossée, qui consacre son article aux médias Roms en France et en Hongrie, montre que le développement remarquable des sites Internet tsiganes (blogs et tout récemment réseaux sociaux) «participe d'un phénomène politique de reconstruction d'une identité commune transnationale malgré la diversité des ancrages et identifications locales ».

Helena Prado analyse la façon dont les médias nikkei contribuent à représenter une identité transnationale nippo-brésilienne construite à la fois sur un mélange d'influences culturelles japonaises et brésiliennes et de représentations sociales. Selon l'auteure, la transformation de la communauté nikkei, devenue « mobile, transnationale et délocalisée », plus que jamais liée à deux pays et deux cultures, a conduit à un changement de la presse et à une diversification des sources médiatiques.

Dans son étude des sites Internet des populations noires, Souley Hassane montre que l'augmentation des interconnexions à l'échelle mondiale permet notamment de manifester des appartenances communes en faisant appel à un imaginaire historique réinterprétant les traditions africaines. Cependant ces sites s'adressent en grande majorité aux communautés noires, locales et nationales.

L'objectif poursuivi dans la publication des articles consacrés aux médias des minorités ethniques était de donner quelques réponses à la question de savoir quelles sont les stratégies de représentation des groupes ethniques d'une part et d'autre part comment les différentes identités culturelles, religieuses, politiques, linguistiques se recomposent de façon complexe. Ils montrent non seulement que les médias sont des 
espaces éditoriaux privilégiés de construction identitaire pour les minorités ethniques, mais aussi des espaces d'expressions contradictoires des différents « entrepreneurs » d'identifications. Avec le développement des possibilités d'accès à l'Internet, la facilité de créer des blogs, d'ouvrir des forums, de faire vivre une presse électronique, de podcaster des émissions, la question qu'il convient de continuer à explorer est de savoir comment s'expriment sur le Net des identités multiples dans ce qu'il est convenu aujourd'hui d'appeler «l'espace mondialisé ».

\section{Références bibliographiques}

ALLARD Laurence (2005) Express Yourself 2.0! Blogs, pages perso, fansubbing... De quelques agrégats technoculturels ordinaires à l'âge de l'expressivisme généralisé, in Éric Maigret, Éric Macé (éds.), Penser les médiacultures. Nouvelles pratiques et nouvelles approches de la représentation du monde, Paris, Armand Colin/INA.

BADIE Bertrand (1995) La fin des territoires. Essai sur le désordre international et sur l'utilité sociale du respect, Paris, Fayard.

BAUMAN Zygmunt (2000) Liquid Modernity, Cambridge, Polity Press.

BECK Ulrich (1992) Risk Society: Towards a New Modernity, London, Sage.

BEN AMOR-MATHIEU Leïla (2000) Les télévisions hispaniques aux États-Unis. L'invention d'une communauté, Paris, CNRS Éditions.

BLONDEAU Loïc, avec Laurence ALLARD (2007) Devenir média. L'activisme sur Internet, entre défection et expérimentation, Paris, Éd. Amsterdam.

CHARON Jean-Marie (2003) Les médias en France, Paris, La Découverte, « Repères ».

COTTLE Simon (ed.) (2001) Ethnic Minorities and the Media, Maidenhead, Open University Press.

DIMINESCU Dana (2007) Le migrant connecté. Pour un manifeste épistémologique, Migrations Société, 17(102), pp. 275-292.

DOWNING John, HUSBAND Charles (2005) Representing 'Race'. Racisms, Ethnicities and Media, London, Sage.

GEORGIOU Myria, GUEDES-BAILEY Olga, HARINDRANATH Ramaswani (eds.) (2007) Transnational Lives and the Media: Reimagining Diasporas, Basingstoke, Palgrave.

GEORGIOU Myria (2006) Diaspora, Identity and the Media: Diasporic Transnationalism and Mediated Spatialities, New York, Hampton Press.

GIDDENS Anthony (1996) Affluence, Poverty and the Idea of a Post-Scarcity Society, Development and Change, 27(2), pp. 365-377.

GRANOVETTER Mark (1973) The Strenght of Weak Ties, American Journal of Sociology, 78(6), pp. 1360-1380.

GUAAYBESS Tourya (2005) Télévisions arabes sur orbite : un système médiatique en mutation, 1960-2004, Paris, CNRS Éditions.

HALL Stuart (1999) Thinking the Diaspora. Home-Thoughts from Abroad, Small Axe, 3(6), pp. 1-18.

HASSANE Souley (2009) L'archivage de l'Internet et la formation d'une mémoire numérique mondiale : enjeux et perspectives du web migrant, E-Migrinter, dossier « Documentation sur Internet et migrations internationales $», \mathrm{n}^{\circ} 3$.

HUSBAND Charles (2001) Media and the Public Sphere in Multi-Ethnic Societies, in Simon Cottle (ed.), Ethnic Minorities and the Media, Maidenhead, Open University Press. 
HUSBAND Charles (1994) A Richer Vision. The Development of Ethnic Minority Media in Western Democracies, Paris/London, Unesco/John Libbey.

LAMLOUM Olfa (2004) Al-Jazira, miroir rebelle et ambigu du monde arabe, Paris, La Découverte.

LICOPPE Christian (2002) Sociabilité et technologies de communication. Deux modalités d'entretien des liens interpersonnels dans le contexte du déploiement des dispositifs de communication mobiles, Réseaux, $\mathrm{n}^{\circ} 112-113$, pp. 172-210.

MARTHOZ Jean-Paul (2001) Médias et «va-et-vient» communicationnel des diasporas, in Reynald Blion, Isabelle Rigoni (éds.), D'un voyage à l'autre. Des voix de l'immigration pour un développement pluriel, Paris, Karthala/Institut Panos Paris.

MATTELART Tristan (éd.) (2009) TIC et diasporas, TIC\&Société, nº spécial, 3(1-2).

MATTELART Tristan (éd.) (2007) Médias, migrations et cultures transnationales, Bruxelles, De Boeck/INA, coll. Médias recherches.

MATTELART Tristan (éd.) (2002) La mondialisation des médias contre la censure. Tiers Monde et audiovisuel sans frontières, Paris/Bruxelles, INA/De Boeck.

MOREAU DEFARGES Philippe (1993) La mondialisation. Vers la fin des territoires ?, Paris, Dunod/IFRI.

NAFICY Hamid (1993) The Making of Exile Cultures. Iranian Television in Los Angeles, Minneapolis, University of Minneapolis Press.

NEDELCU Mihaela (2009) Le migrant online. Nouveaux modèles migratoires à l'ère du numérique, Paris, L'Harmattan, Coll. Questions sociologiques.

PARK Robert Ezra (1970 [1922]) The Immigrant Press and its Control, Westport, Greenwood Press.

PARK Robert Ezra (2008 [1923, 1940, 1944]) Le journaliste et le sociologue, Articles présentés et commentés par Géraldine Muhlmann et Edwy Plenel, Paris, Seuil.

PROULX Serge (2008) Des nomades connectés : vivre ensemble à distance, Hermès, (51), pp. 155-166.

RIGONI Isabelle (2010, à paraître) Les luttes de dénomination autour des « médias des minorités ethniques ». Réflexions méthodologiques et épistémologiques, Dossier « Fixer les mobilités : usages et paradoxes des catégorisations en migration », Migrations Société, $\mathrm{n}^{\circ} 128$, marsavril.

RIGONI Isabelle (2008) France, de la visibilité aux contenus (avec Reynald Blion, Claire Frachon, Catherine Humblot), in Claire Frachon, Virginie Sassoon (éd.), Médias et diversité. De la visibilité aux contenus. État des lieux en France, au Royaume-Uni, en Allemagne et aux États-Unis, Paris, Karthala/Institut Panos Paris.

RIGONI Isabelle (éd.) (2007a) Qui a peur de la télévision en couleurs ? La diversité culturelle dans les médias, Montreuil, Aux Lieux d'Être.

RIGONI Isabelle (2007b) De l'immigration à l'immigré : quand l'objet devient sujet, Migrations Société, «Informer sur les migrations », 19 (111-112), mai-août, pp. 201-214.

SIAPERA Eugenia (2009) Theorizing the Muslim Blogosphere, in A. Russell, N. Echchaibi (eds.), International Blogging: Identity, Politics and Networked Publics, New York, Peter Lang.

SIAPERA Eugenia (2007) Radical Democratic Politics and Online Islam, in L. Dahlberg, E. Siapera (eds.), Radical Democracy and the Internet. Basingstoke, Macmillan Palgrave.

SIAPERA Eugenia (2006a) Islam, the Internet and Multicultural Politics, International Journal of Media and Cultural Politics, 2(3), pp. 331-346.

SIAPERA Eugenia (2006b) Multiculturalism Online: The Internet and the Dilemmas of Multicultural Politics, European Journal of Cultural Studies, 9(1), pp. 5-24.

SIMON Gildas (2008) La planète migratoire dans la mondialisation, Paris, Armand Colin.

SIMON Pierre-Jean (2006) Pour une sociologie des relations interethniques et des minorités, Rennes, Presses universitaires de Rennes. 
TARRIUS Alain (1996) Territoires circulatoires et espaces urbains, Annales de la Recherche Urbaine, $\mathrm{n}^{\circ}$ 59-60, pp. 50-59.

TITLEY Gavan (2008) Media Transnationalism in Ireland: an Examination of Polish Media Practices, Translocations: The Irish Migration, Race and Social Transformation Review, 3(1), pp. 29-49.

WIEVIORKA Michel (éd.) (1996) Une société fragmentée. Le multiculturalisme en débat, Paris, La Découverte. 